Supporting Information

\title{
Customizable Multi-Dimensional Self-Wrinkling Structure Constructed via Modulus Gradient in Chitosan Hydrogels
}

Xiaojuan Lei, ${ }^{[\mathrm{a}]}$ Dongdong Ye, ${ }^{[\mathrm{a}]}$ Jie Chen, ${ }^{[\mathrm{d}]}$ Shan Tang, ${ }^{[\mathrm{d}]}$ Pingchuan Sun, ${ }^{[\mathrm{c}]}$ Lingyun Chen, ${ }^{[\mathrm{e}]}$ Ang $\mathrm{Lu},{ }^{*[\mathrm{a}]}$ Yumin Du, ${ }^{[\mathrm{b}]}$ Lina Zhang*[a]

[a] College of Chemistry and Molecular Sciences, Hubei Engineering Center of Natural Polymer-based Medical Materials, Wuhan University, Wuhan 430072, China.

[b] College of Resource and Environmental Science, Wuhan University, Wuhan 430072, China.

[c] College of Chemistry, Nankai University, Tianjin 300071, China.

[d] Department of Mechanicals, Dalian University of Technology, Dalian 116024, China.

[e] Department of Agricultural, Food and Nutritional Science, University of Alberta, Edmonton, AB, T6G 2P5, Canada.

*E-mail: zhangln@whu.edu.cn (L. Zhang); duyumin@whu.edu.cn (Y. Du); anglu@whu.edu.cn (A. $\mathrm{Lu})$ 
(a)

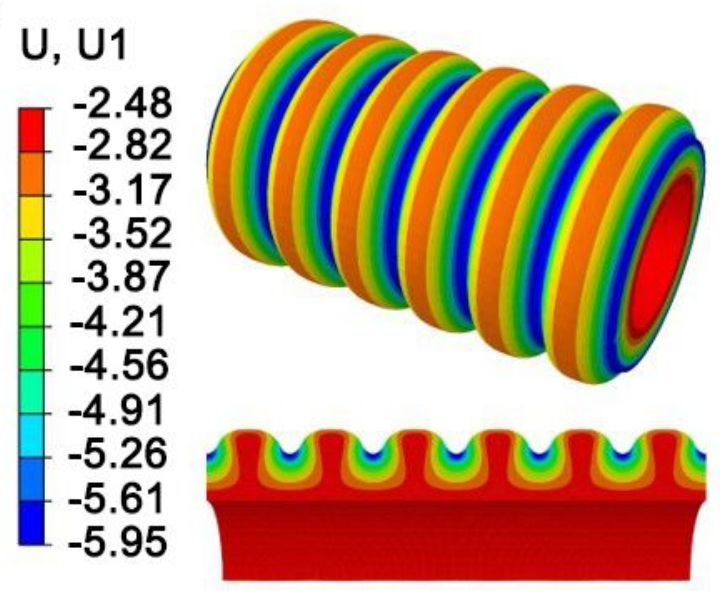

(c) $\mathrm{U}, \mathrm{U} 1$

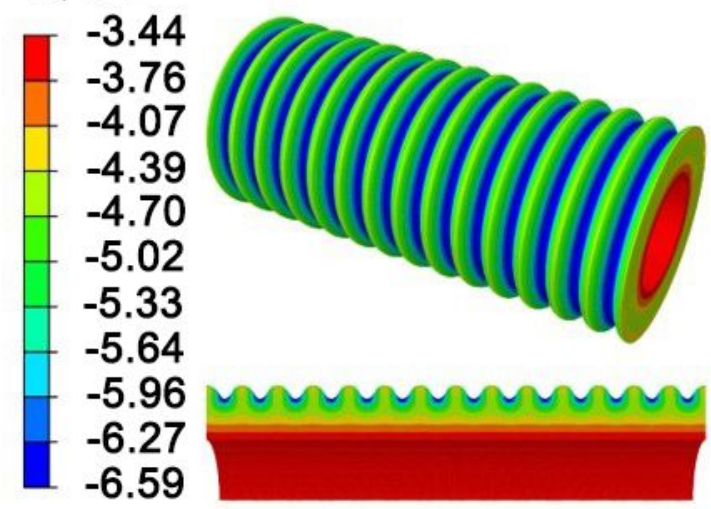

(b)

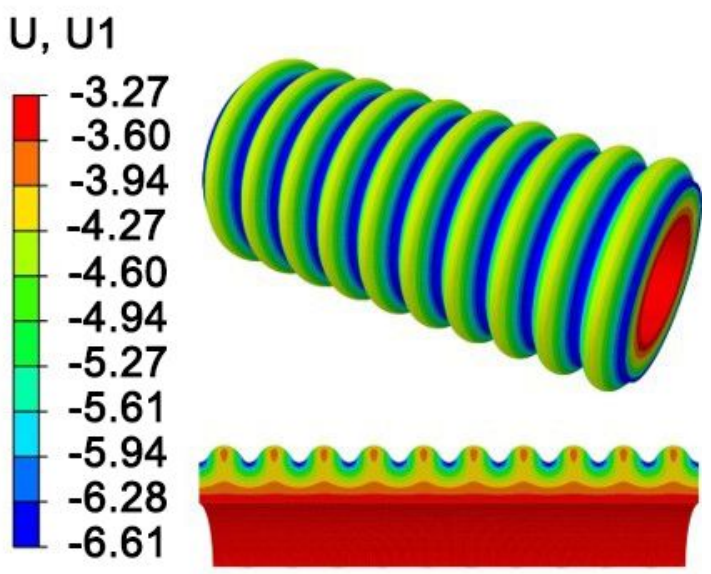

(d) U, U1

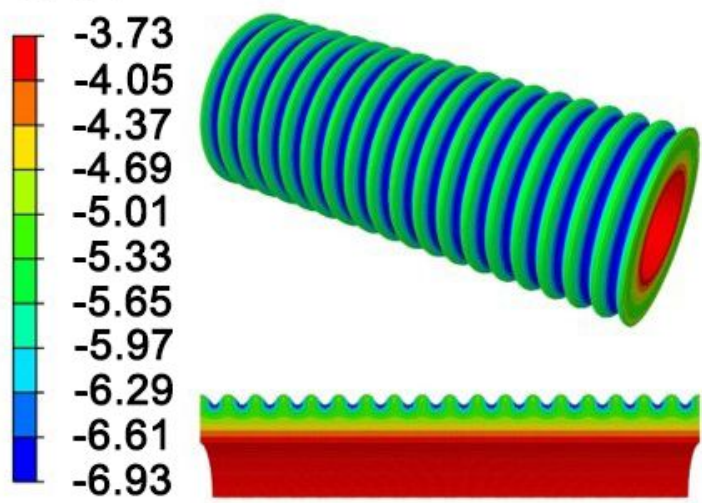

Fig. S1. Finite-element model of the self-wrinkling deformation of a tubular gel. 


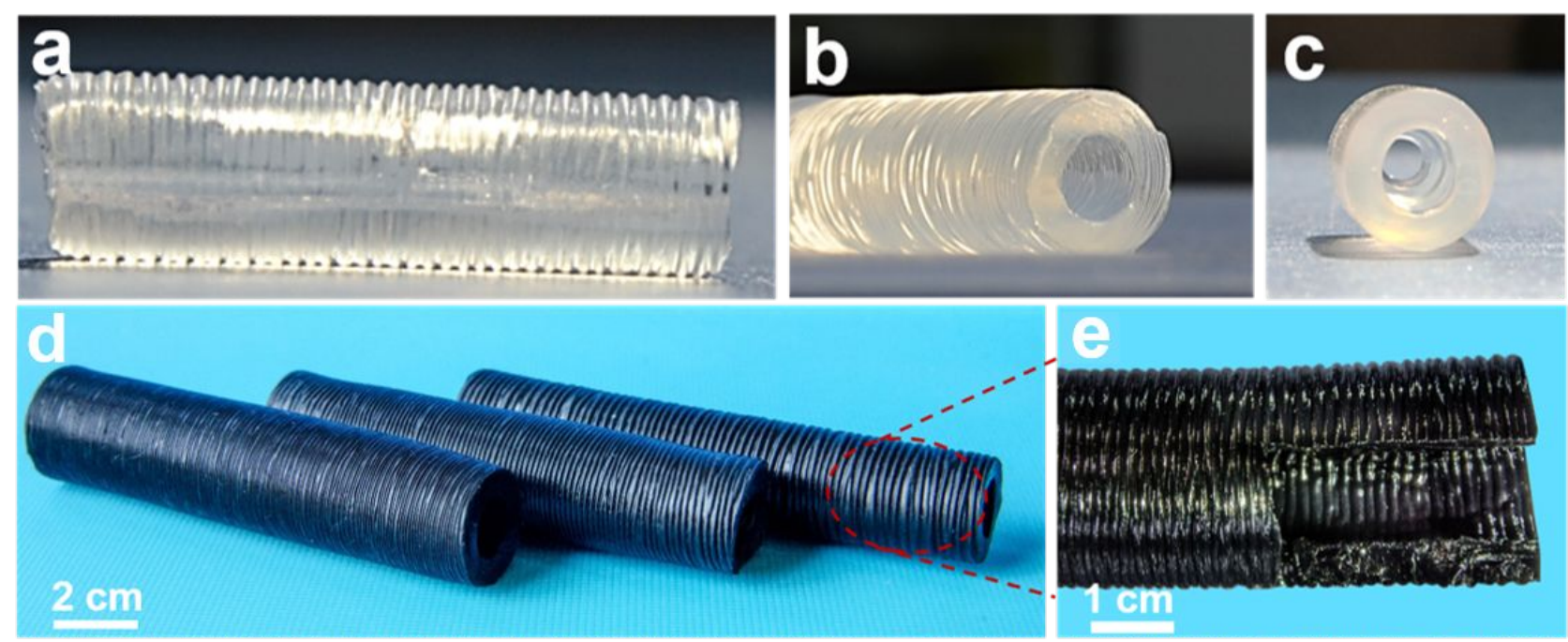

Fig. S2. Photos of the self-wrinkling gels (a-c) and conductive self-wrinkling gels (de). 
Movie 1. Fabrication of the self-wrinkling hydrogel by water bath treatment.

Movie 2. Compressibility displays of the composite self-wrinkling gel in different directions. 